\title{
HYBRID DELAY EVOLUTION SYSTEMS WITH NONLINEAR CONSTRAINTS
}

\author{
OCTAVIA-MARIA BOLOJAN ${ }^{1}$ AND RADU PRECUP ${ }^{2}$ \\ ${ }^{1,2}$ Department of Mathematics \\ Babeş-Bolyai University \\ 400084, Cluj-Napoca, ROMANIA \\ ${ }^{1}$ Department of Computers and Information Technology \\ University of Oradea \\ 410087, Oradea, ROMANIA
}

\begin{abstract}
Motivated by the importance of reaction-diffusion systems in modeling real processes with memory, we are interested in the existence of mild solutions for systems of abstract delay evolution equations subjected to general nonlinear constraints. Wishing to allow the system nonlinearities to behave independently as much as possible, we use a vector approach based on matrices, vector-valued norms and a vector version of Krasnoselskii's fixed point theorem for a sum of two operators. The hybrid character of the systems comes from the different nature of the metrical and topological conditions imposed to the component equations. Also, the assumptions are put in connection with the support of the nonlinear constraints. Two examples are given to illustrate the theory.
\end{abstract}

AMS Subject Classification: 34K30, 35K90, 47J35

Key Words: nonlinear evolution equation, nonlocal initial condition, delay, Krasnoselskii's fixed point theorem for a sum of operators

Received: October 30, 2016; Accepted: May 23, 2018;

Published: September 15, $2018 \quad$ doi: $\quad 10.12732 /$ dsa.v27i4.6

Dynamic Publishers, Inc., Acad. Publishers, Ltd. https://acadsol.eu/dsa

\section{INTRODUCTION}

In this paper, we are concerned with the existence of solutions to the semilinear system of abstract delay evolution equations with constraints (nonlocal initial conditions), of 
the type

$$
\begin{cases}u_{i}^{\prime}(t)+A_{i} u_{i}(t)=F_{i}\left(t, u_{t}\right), & t \in[0, T] \\ g_{i}(u)(t)=0, & t \in[-\tau, 0] \\ & i=1, \ldots, n\end{cases}
$$

Here $n \geq 1$, and for each $i \in J:=\{1, \ldots, n\}$, the linear operator $-A_{i}: D\left(A_{i}\right) \subseteq$ $X_{i} \rightarrow X_{i}$ generates a $C_{0}$-semigroup of contractions $\left\{S_{i}(t) ; t \geq 0\right\}$ on a Banach space $\left(X_{i},|\cdot|_{X_{i}}\right), \tau \geq 0, u \in C([-\tau, T], X)$, where $X=X_{1} \times \ldots \times X_{n}$, and for each $t, u_{t}$ is the restriction of $u$ to $[t-\tau, t]$ shifted to the interval $[-\tau, 0]$, i.e., $u_{t} \in C([-\tau, 0], X)$ and

$$
u_{t}(s)=u(t+s), \quad s \in[-\tau, 0] .
$$

The nonlinear perturbations in equations are given by the continuous mappings $F_{i}$ from $[0, T] \times C([-\tau, 0], X)$ to $X_{i}$, and the constraints are expressed by the continuous mappings $g_{i}$ from $C([-\tau, T], X)$ to $C([-\tau, 0], X)$.

Here are some particular cases of equations that could appear in system (1.1):

(E1) If $\tau=0$, then $u_{t}$ reduces to $u(t)$ and the equations of the system are without delay, of the form

$$
u_{i}^{\prime}(t)+A_{i} u_{i}(t)=F_{i}(t, u(t)) .
$$

(E2) The following delay equations

$$
u_{i}^{\prime}(t)+A_{i} u_{i}(t)=G_{i}\left(t, u(t), u_{1}\left(t-\gamma_{1}\right), \ldots, u_{n}\left(t-\gamma_{n}\right)\right),
$$

where $\gamma_{1}, \ldots, \gamma_{n} \geq 0$ are given numbers, are particular cases of the equations from (1.1). In this case $\tau=\max \left\{\gamma_{1}, \ldots, \gamma_{n}\right\}$ and the delays are discretely distributed in the interval $[0, \tau]$.

(E3) It may happen that the delays $\gamma_{1}, \ldots, \gamma_{n}$ in (1.3) are continuous nonnegative functions of time, so that (1.3) takes the form

$$
u_{i}^{\prime}(t)+A_{i} u_{i}(t)=G_{i}\left(t, u(t), u_{1}\left(t-\gamma_{1}(t)\right), \ldots, u_{n}\left(t-\gamma_{n}(t)\right)\right) .
$$

Then $\tau=\max \left\{\left|\gamma_{1}\right|_{C[0, T]}, \ldots,\left|\gamma_{n}\right|_{C[0, T]}\right\}$ and the delays are time-depending discretely distributed in $[0, \tau]$.

(E4) The general form of the system (1.1) also covers, as a particular case, the integro-differential systems whose equations are of the form

$u_{i}^{\prime}(t)+A_{i} u_{i}(t)=G_{i}\left(t, u(t), \int_{0}^{\tau} h_{1}\left(t, s, u_{1}(t-s)\right) d s, \ldots, \int_{0}^{\tau} h_{n}\left(t, s, u_{n}(t-s)\right) d s\right)$, with the delays continuously distributed on $[0, \tau]$.

As concerns the constraints in system (1.1), let us mention some particular cases: (C1) Initial condition:

$$
u_{0}=\varphi \text {, }
$$


where $\varphi \in C([-\tau, 0], X)$ is given. Explicitly, this means $u(s)=\varphi(s)$ for every $s \in[-\tau, 0]$. In this case $g(u)=\varphi-u_{0}$.

(C2) Linear multi-point conditions (linear nonlocal initial conditions of discrete type):

$$
u_{i}(s)=\varphi_{i}(s)+\sum_{j=1}^{m_{i}} a_{i j}(s) u_{i}\left(s+t_{i j}\right), \quad s \in[-\tau, 0], i=1, \ldots, n,
$$

where $0<t_{i j}<t_{i, j+1} \leq T$ for $j=1, \ldots, m_{i}$ and $i=1, \ldots, n$. In this case $g_{i}(u)(s)=$ $\varphi_{i}(s)-u_{i}(s)+\sum_{j=1}^{m_{i}} a_{i j}(s) u_{i}\left(s+t_{i j}\right)$. These conditions include in particular the initial condition, and the periodicity condition

$$
u_{0}=u_{T}
$$

which explicitly means that $u(s)=u(T+s)$ for every $s \in[-\tau, 0]$.

Physically, if $\tau=0$ and $u(t)$ stands for the state at time $t$ of a process taking place in a spatial domain, being a function of space variable $x$, i.e., $u(t)(x)=u(x, t)$, the periodic condition requires that the final state of the process coincides with the initial state, i.e., $u(x, T)=u(x, 0)$. However, due to stimulatory or inhibitory exterior sources, one may expect an increased or a decreased final state, that is

$$
u(x, T)=\omega(x) u(x, 0)
$$

with $\omega(x) \geq 1$, or $\omega(x) \leq 1$, respectively. Such kind of conditions were considered in [24], and are also covered by (1.4).

(C3) Linear nonlocal initial conditions of continuous type, given by integrals:

$$
u_{0}=\varphi+\int_{0}^{T} k(\cdot, t) u_{t} d t
$$

or explicitly

$$
\begin{aligned}
u_{i}(s) & =\varphi_{i}(s)+\int_{0}^{T} k_{i}(s, t) u_{i}(t+s) d t \\
& =\varphi_{i}(s)+\int_{s}^{T+s} k_{i}(s, t-s) u_{i}(t) d t, \quad s \in[-\tau, 0] .
\end{aligned}
$$

For $\tau=0$, this condition reduces to

$$
u_{i}(0)=\varphi_{i}^{0}+\int_{0}^{T} k_{i}(t) u_{i}(t) d t
$$

and we can understand it as in [33], assuming that the concentration of some diffusing substance whose initial level is unspecified, is balanced against some weighted average of all levels of concentration along the time interval $[0, T]$. 
Since Volterra's pioneering works on integro-differential equations with delayed effects in population dynamics and materials with memory, the theory of delay differential equations progressed dramatically stimulated by the development of functional analysis and its numerous real world applications, wherever (in physics, chemistry, biology, medicine, economy etc, see e.g., [25]) the evolution of a process depends on its history in an essential way.

Differential equations with general boundary conditions of multi-point or integral type, have a long history (see, e.g. Cioranescu [16], Whyburn [46], Conti [17]), and have gained a special attention in the last decades motivated by concrete applications in different domains. See, for example, [2], [3], [5], [6], [8], [19], [23], [30], [31], [36], [39], [45], the recent survey paper [40] and the references therein.

For parabolic problems with nonlocal initial conditions we mention the papers of Kerefov [24], Vabishchevich [41], Chabrowski [14], Deng [18], Pao [37], Olmstead and Roberts [33], and Chapter 10 in [28], where nonlocal versions of some deterministic models from physics, mechanics, biology and medicine are stated. Abstract evolution equations with nonlocal initial conditions were considered by Byszewski [12], Jackson [22], Lin and Liu [26]. For more recent contributions, we refer the readers to the papers [4], [7], [9], [10], [13], [21], [26], [27], [29], [32], [35], [44] and the very recent monograph [11].

Throughout the paper, by $[-\tau, a]$ we shall denote the support of the constraints, that is the smallest subinterval $[-\tau, a]$ with $0 \leq a \leq T$ such that

$$
\begin{aligned}
g_{i}(u) & =g_{i}(v), \quad i=1, \ldots, n \\
\text { for every } u, v & \in C([-\tau, T], X) \text { with }\left.u\right|_{[-\tau, a]}=\left.v\right|_{[-\tau, a]} .
\end{aligned}
$$

Here by $\left.u\right|_{[-\tau, a]}$ we mean the restriction of the function $u$ to the interval $[-\tau, a]$.

The notion of support plays an essential role in the existence results for nonlocal problems, as first shown in the papers [3] and [4]. More exactly, in these papers it is shown that stronger conditions on nonlinearities have to be asked on the support subinterval, compared to those required on the rest of the interval on which the problem is considered. Mathematically, the integral equation equivalent to the nonlocal problem is of Fredholm type on the support interval, and of Volterra type on the rest of the interval. From a physical point of view, the evolution of a process is subjected to some constraints until a given moment of time, and becomes free of any constraints after that moment.

This double nature Fredholm-Volterra of the nonlocal problems, due to the support of the nonlocal conditions, makes useful to consider a split norm on the functional space where the problem is investigated. Thus, for the delay system (1.1), we shall consider here for the first time the split norm on $C\left([-\tau, T], X_{i}\right)$, defined by 


$$
|u|_{\tau}=\max \left\{|u|_{C\left([-\tau, a], X_{i}\right)}, \quad|u|_{C_{\theta}\left([a-\tau, T], X_{i}\right)}\right\}
$$

where $|u|_{C\left([-\tau, a], X_{i}\right)}$ is the usual max norm

$$
|u|_{C\left([-\tau, a], X_{i}\right)}=\max _{t \in[-\tau, a]}|u(t)|_{X_{i}}
$$

while for any $\theta>0,|u|_{C_{\theta}\left([a-\tau, T], X_{i}\right)}$ is the Bielecki type norm on $C\left([a-\tau, T], X_{i}\right)$,

$$
|u|_{C_{\theta}\left([a-\tau, T], X_{i}\right)}=\max _{t \in[a, T]}\left(\left|u_{t}\right|_{C\left([-\tau, 0], X_{i}\right)} e^{-\theta(t-a)}\right) .
$$

In the particular case of equations without delay, when $\tau=0$, the norm (1.5) reduces to the split norm that we already considered in our previous papers [3]-[5], [30] and [31].

As we shall see in the sequel, the hybrid character of the system comes from the different compactness properties associated to the equations of the system. The system will be split into to subsystems: the first $m$ equations, and the last $n-$ $m$ equations $(0 \leq m \leq n)$, and two existence results will be proved. The first one assumes on $g_{i}$, Lipschitz condition for $i=1, \ldots, m$, and completely continuity and some growth condition for $i=m+1, \ldots, n$; the second result requires the Lipschitz condition for all the mappings $g_{i}(i=1, \ldots, n)$. Both results require for $i=1, \ldots, m$, that the operator $-A_{i}$ generate a $C_{0}$-semigroup of contractions, and the mapping $F_{i}$ satisfies a Lipschitz condition, while for $i=m+1, \ldots, n$, that $-A_{i}$ generates a compact $C_{0}$-semigroup of contractions, and $F_{i}$ satisfies only a growth condition. The proof of these two results is based on a vector version of Krasnoselskii's fixed point theorem for a sum of a compact map and a generalized contraction in Perov's sense.

\section{PRELIMINARIES}

For the treatment of systems we use the vector approach based on vector-valued metrics and norms, and matrices instead of constants.

Let us make the convention that the elements of $\mathbb{R}^{n}$ are seen as column vectors. By a vector-valued metric on a set $Y$ we mean a mapping $d: Y \times Y \rightarrow \mathbb{R}_{+}^{n}$ such that $d(x, y)=0$ if and only if $x=y ; d(x, y)=d(y, x)$ for all $x, y \in Y$ and $d(x, y) \leq$ $d(x, z)+d(z, y)$ for all $x, y, z \in Y$. Here by $\leq$ we mean the natural componentwise order relation of $\mathbb{R}^{n}$, more exactly, if $r, s \in \mathbb{R}^{n}, r=\left(r_{1}, \ldots, r_{n}\right), s=\left(s_{1}, \ldots, s_{n}\right)$, then by $r \leq s$ one means that $r_{i} \leq s_{i}$ for $i=1, \ldots, n$. A set $Y$ together with a vector-valued metric $d$ is called a generalized metric space. For such a space, the notions of Cauchy sequence, convergence, completeness, open and closed set, are similar to those in usual metric spaces. 
Similarly, a vector-valued norm on a linear space $Y$, is defined as being a mapping $\|\cdot\|: Y \rightarrow \mathbb{R}_{+}^{n}$ with $\|x\|=0$ only for $x=0 ;\|\lambda x\|=|\lambda|\|x\|$ for $x \in Y, \lambda \in \mathbb{R}$, and $\|x+y\| \leq\|x\|+\|y\|$ for every $x, y \in Y$. To any vector-valued norm $\|\cdot\|$ one can associate the vector-valued metric $d(x, y):=\|x-y\|$. A linear space $Y$ endowed with a vector-valued norm $\|\cdot\|$ is called a generalized Banach space if $Y$ is complete with respect to the associated vector-valued metric $d$.

If $(Y, d)$ is a generalized metric space with $d$ taking values in $\mathbb{R}^{n}$, we say that a mapping $N: Y \rightarrow Y$ is a generalized contraction (in Perov's sense) if there exists a square matrix $M$ of size $n$ with nonnegative entries such that its powers $M^{k}$ tend to the zero matrix 0 as $k \rightarrow \infty$, and

$$
d(N(x), N(y)) \leq M d(x, y) \text { for all } x, y \in Y
$$

Such a matrix is said to be a Lipschitz matrix. For such type of mappings, the following generalization of Banach's contraction principle holds.

Theorem 2.1 (Perov). If $(Y, d)$ is a complete generalized metric space, then any generalized contraction $N: Y \rightarrow Y$ with the Lipschitz matrix $M$ has a unique fixed point $x^{*}$, and

$$
d\left(N^{k}(x), x^{*}\right) \leq M^{k}(I-M)^{-1} d(x, N(x)),
$$

for all $x \in Y$ and $k \in \mathbb{N}$ (where $I$ stands for the identity matrix of the same size as $M)$.

In this paper we use the following generalization of Theorem 2.1, a vector version of Krasnoselskii's fixed point theorem for a sum of two operators, due to Viorel [42], whose proof is sketched below for the reader's convenience.

Theorem 2.2. Let $(Y,\|\cdot\|)$ be a generalized Banach space, $D \subset Y$ a nonempty bounded closed convex set and $N: D \rightarrow Y$ a mapping such that

(i) $N=A+B$ with $A: D \rightarrow Y$ a generalized contraction in Perov's sense, and $B: D \rightarrow Y$ a compact operator;

(ii) $A(u)+B(v) \in D$ for every $u, v \in D$.

Then $N$ has at least one fixed point in $D$.

Proof. For any fixed $v \in D$, the operator $u \in D \mapsto A(u)+B(v)$ is a generalized contraction from $D$ to $D$, which by Perov's theorem has a unique fixed point $u_{v}$ in $D$. Thus we have defined the operator $S: D \rightarrow D, S(v)=u_{v}$. For any two elements $v_{1}, v_{2} \in D$, from

$$
S\left(v_{1}\right)=A\left(S\left(v_{1}\right)\right)+B\left(v_{1}\right), \quad S\left(v_{2}\right)=A\left(S\left(v_{2}\right)\right)+B\left(v_{2}\right),
$$


since $A$ is a generalized contraction, we deduce that

$$
\left\|S\left(v_{1}\right)-S\left(v_{2}\right)\right\| \leq(I-M)^{-1}\left\|B\left(v_{1}\right)-B\left(v_{2}\right)\right\|,
$$

where $M$ is the Lipschitz matrix of $A$. From this inequality, the continuity of $S$ is obvious. To show that $S(D)$ is relatively compact, it is enough to prove that for any sequence $\left(v_{k}\right)$ of elements from $D$, there exists a convergent subsequence of $\left(S\left(v_{k}\right)\right)$. Since $B$ is compact, there is a convergent subsequence of $\left(B\left(v_{k}\right)\right)$, still denoted by $\left(B\left(v_{k}\right)\right)$ for simplicity. Then from $(2.1)$,

$$
\left\|S\left(v_{k}\right)-S\left(v_{k+p}\right)\right\| \leq(I-M)^{-1}\left\|B\left(v_{k}\right)-B\left(v_{k+p}\right)\right\|,
$$

which shows that $\left(S\left(v_{k}\right)\right)$ is a Cauchy sequence. Therefore $S$ is compact. Then Schauder's fixed point theorem implies that $S$ has at least one fixed point in $D$, which is also a fixed point of $N$.

There are known several characterizations of matrices like that in Perov's theorem (see, e.g., [1] and [38]). More exactly, for a square matrix $M$ of size $n$ with nonnegative entries, i.e., $M \in \mathcal{M}_{n \times n}\left(\mathbb{R}_{+}\right)$, the following statements are equivalent:

(a) $M^{k} \rightarrow 0$ as $k \rightarrow \infty$;

(b) $I-M$ is nonsingular and $(I-M)^{-1}=I+M+M^{2}+\ldots$;

(c) the eigenvalues of $M$ are located inside the unit disc of the complex plane, i.e., $\rho(M)<1$, where $\rho(M)$ is the spectral radius of $M$;

(d) $I-M$ is nonsingular and inverse-positive, i.e., $(I-M)^{-1}$ has nonnegative entries.

The following two obvious propositions will be used in the proof of the main result:

Proposition 2.3. If $M \in \mathcal{M}_{n \times n}\left(\mathbb{R}_{+}\right)$is a matrix with $\rho(M)<1$, then $\rho(\widetilde{M})<1$ for every matrix $\widetilde{M} \in \mathcal{M}_{n \times n}\left(\mathbb{R}_{+}\right)$whose elements are close enough to the corresponding elements of $M$.

Proposition 2.4. If $M \in \mathcal{M}_{n \times n}\left(\mathbb{R}_{+}\right)$is a matrix with $\rho(M)<1$, then $\rho(\widehat{M})<1$ for every matrix $\widehat{M} \in \mathcal{M}_{n \times n}\left(\mathbb{R}_{+}\right)$such that $\widehat{M} \leq M$ componentwise.

The role of matrices with spectral radius less than one in the study of operator systems was pointed out in [38], in connection with several abstract principles from nonlinear functional analysis.

Basic notions and results from the semigroup theory that are frequently used in the present work can be found, for example, in [15], [20] and [43]. 


\section{MAIN RESULTS}

It is convenient that the constraints in problem (1.1) are written in the equivalent form

$$
u_{i}(t)=\alpha_{i}(u)(t), \quad t \in[-\tau, 0],
$$

where $\alpha_{i}(u)=g_{i}(u)+\left(u_{i}\right)_{0}$. The meaning of the notation $\left(u_{i}\right)_{0}$ is that given by (1.2), i.e., $\left(u_{i}\right)_{0} \in C\left([-\tau, 0], X_{i}\right),\left(u_{i}\right)_{0}(s)=u_{i}(0+s)=u_{i}(s)$ for $s \in[-\tau, 0]$. In what follows the conditions on the constraints will be given in terms of the mappings $\alpha_{i}: C\left([-\tau, T], X_{i}\right) \rightarrow C\left([-\tau, 0], X_{i}\right)$.

Looking for mild solutions to the problem (1.1), we are led in a standard way to the following integral system $C([-\tau, T], X)$,

$$
\begin{cases}u_{i}(t)=\alpha_{i}(u)(t), & t \in[-\tau, 0], \\ u_{i}(t)=S_{i}(t) \alpha_{i}(u)(0)+\int_{0}^{t} S_{i}(t-s) F_{i}\left(s, u_{s}\right) d s, & t \in[0, T], \\ & i=1, \ldots, n\end{cases}
$$

We first give a list of conditions in terms of the mappings $A_{i}, F_{i}$ and $\alpha_{i}$ and of a number $p>1$ :

(H1) The linear operator $-A_{i}: D\left(A_{i}\right) \subseteq X_{i} \rightarrow X_{i}$ generates a $C_{0}$-semigroup of contractions on the Banach space $X_{i}$.

(H1*) The linear operator $-A_{i}: D\left(A_{i}\right) \subseteq X_{i} \rightarrow X_{i}$ generates a compact $C_{0^{-}}$ semigroup of contractions on the Banach space $X_{i}$.

(H2) The mapping $F_{i}:[0, T] \times C([-\tau, 0], X) \rightarrow X_{i}$ is continuous and there exist $a_{i j} \in L^{1}\left(0, T ; \mathbb{R}_{+}\right)$with $\left.a_{i j}\right|_{[0, a]} \in L^{p}(0, a)$, and $c_{i} \in C\left([0, T], \mathbb{R}_{+}\right)$for $j \in J$, such that

$$
\left|F_{i}(t, u)\right|_{X_{i}} \leq \sum_{j=1}^{n} a_{i j}(t)\left|u_{j}\right|_{C\left([-\tau, 0], X_{j}\right)}+c_{i}(t)
$$

for all $u \in C([-\tau, 0], X)$ and $t \in[0, T]$.

(H2*) The mapping $F_{i}:[0, T] \times C([-\tau, 0], X) \rightarrow X_{i}$ is continuous and there exists $a_{i j} \in L^{1}\left(0, T ; \mathbb{R}_{+}\right)$with $\left.a_{i j}\right|_{[0, a]} \in L^{p}(0, a)$ for every $j \in J$, such that

$$
\left|F_{i}(t, u)-F_{i}(t, v)\right|_{X_{i}} \leq \sum_{j=1}^{n} a_{i j}(t)\left|u_{j}-v_{j}\right|_{C\left([-\tau, 0], X_{j}\right)}
$$

for $u, v \in C([-\tau, 0], X)$ and $t \in[0, T]$. 
(H3) The mapping $\alpha_{i}: C([-\tau, T], X) \rightarrow C\left([-\tau, 0], X_{i}\right)$ is completely continuous and there exist $b_{i j} \in \mathbb{R}_{+}$and $d_{i} \in \mathbb{R}_{+}$for every $j \in J$, such that

$$
\left|\alpha_{i}(u)\right|_{C\left([-\tau, 0], X_{i}\right)} \leq \sum_{j=1}^{n} b_{i j}\left|u_{j}\right|_{C\left([-\tau, a], X_{j}\right)}+d_{i}
$$

for all $u \in C([-\tau, T], X)$.

(H3*) There exist $b_{i j} \in \mathbb{R}_{+}$for every $j \in J$, such that

$$
\left|\alpha_{i}(u)-\alpha_{i}(v)\right|_{C\left([-\tau, 0], X_{i}\right)} \leq \sum_{j=1}^{n} b_{i j}\left|u_{j}-v_{j}\right|_{C\left([-\tau, a], X_{j}\right)}
$$

for all $u, v \in C([-\tau, T], X)$.

Our assumptions will be given differently on two sets of indices,

$$
J_{1}:=\{1, \ldots, m\} \quad \text { and } \quad J_{2}:=\{m+1, \ldots, n\}
$$

where $0 \leq m \leq n$, and it is understood that $J_{1}=\emptyset$ if $m=0$, and $J_{2}=\emptyset$ if $m=n$.

Theorem 3.1. Assume that the conditions (H1), $(\mathrm{H} 2 *)$ and $\left(\mathrm{H} 3^{*}\right)$ hold for every $i \in J_{1}$, and that $\left(\mathrm{H} 1^{*}\right),(\mathrm{H} 2)$ and $(\mathrm{H} 3)$ hold for every $i \in J_{2}$. In addition assume that the spectral radius of the $n \times n$ square matrix $M=\left[m_{i j}\right]$, where

$$
m_{i j}:=\left|a_{i j}\right|_{L^{1}(0, a)}+b_{i j}
$$

is less than one. Then the problem (1.1) has at least one mild solution on $[-\tau, T]$. In case that $m=n$, the solution is unique.

Proof. The integral system (3.1) can be seen as a fixed point equation $u=N(u)$ in $C([-\tau, T], X)$ for the nonlinear operator $N$ from the space $C([-\tau, T], X)$ to itself, $N=\left(N_{1}, \ldots, N_{n}\right)$, where $N_{i}: C([-\tau, T], X) \rightarrow C\left([-\tau, T], X_{i}\right)$ are defined by

$$
N_{i}(u)(t)= \begin{cases}\alpha_{i}(u)(t), & t \in[-\tau, 0], \\ S_{i}(t) \alpha_{i}(u)(0)+\int_{0}^{t} S_{i}(t-s) F_{i}\left(s, u_{s}\right) d s, & t \in[0, T] .\end{cases}
$$

Clearly, the operator $N$ admits the representation $N=A+B$, where

$$
A=\left(N_{1}, \ldots, N_{m}, 0, \ldots, 0\right), \quad B=\left(0, \ldots, 0, N_{m+1}, \ldots, N_{n}\right) .
$$

We shall apply the vector version of Krasnoselskii's fixed point theorem to the operator $N$ on the space

$$
C([-\tau, T], X)=C\left([-\tau, T], X_{1}\right) \times \ldots \times C\left([-\tau, T], X_{n}\right)
$$


endowed with the vector-valued norm

$$
\|u\|=\left(\left|u_{1}\right|_{\tau}, \ldots,\left|u_{n}\right|_{\tau}\right)^{t r}
$$

where for each $i$, by $\left|u_{i}\right|_{\tau}$ we mean the norm in $C\left([-\tau, T], X_{i}\right)$ given by (1.5), with $\theta>0$ chosen below. The result will follow from Theorem 2.2 once the following lemmas have been proved:

Lemma 3.2. There exist $R_{1}, \ldots, R_{n} \geq 0$ such that $A(u)+B(v) \in D$ for every $u, v \in D$, where

$$
D=\left\{u=\left(u_{1}, \ldots, u_{n}\right) \in C([-\tau, T], X):\left|u_{i}\right|_{\tau} \leq R_{i} \text { for } i \in J\right\} .
$$

Lemma 3.3. The operator $A$ is a generalized contraction in Perov's sense on $C([-\tau, T], X)$.

Lemma 3.4. The operator $B$ is completely continuous on $C([-\tau, T], X)$.

Proof of Lemma 3.2. The fact that $A(u)+B(v) \in D$ for every $u, v \in D$ is equivalent to the inequalities

$$
\left|N_{i}(u)\right|_{\tau} \leq R_{i} \quad \text { for } \quad u \in D, i \in J
$$

First note that from (3.3) for $v=0$,

$$
\left|F_{i}(t, u)\right|_{X_{i}} \leq \sum_{j=1}^{n} a_{i j}(t)\left|u_{j}\right|_{C\left([-\tau, 0], X_{j}\right)}+\left|F_{i}(t, 0)\right|_{X_{i}} .
$$

Hence the condition (3.2) also holds for $i \in J_{1}$, with $c_{i}(t)=\left|F_{i}(t, 0)\right|_{X_{i}}$. Similarly, from (3.5), (3.4) also holds for $i \in J_{1}$, with $d_{i}=\left|\alpha_{i}(0)\right|_{C\left([-\tau, 0], X_{i}\right)}$.

In order to prove (3.7), take any $u \in D$ and $i \in J$. For $t \in[-\tau, 0]$,

$$
\left|N_{i}(u)(t)\right|_{X_{i}}=\left|\alpha_{i}(u)(t)\right|_{X_{i}} \leq\left|\alpha_{i}(u)\right|_{C\left([-\tau, 0], X_{i}\right)} \leq \sum_{j=1}^{n} b_{i j}\left|u_{j}\right|_{C\left([-\tau, a], X_{j}\right)}+d_{i} .
$$

For $t \in[0, a]$, since the semigroups are of contractions,

$$
\left|N_{i}(u)(t)\right|_{X_{i}} \leq\left|\alpha_{i}(u)(0)\right|_{X_{i}}+\int_{0}^{t}\left|F_{i}\left(s, u_{s}\right)\right|_{X_{i}} d s .
$$

The term $\left|\alpha_{i}(u)(0)\right|_{X_{i}}$ is evaluated as in (3.8), while for the integral we have

$$
\begin{aligned}
\int_{0}^{t}\left|F_{i}\left(s, u_{s}\right)\right|_{X_{i}} d s & \leq \int_{0}^{t}\left(\sum_{j=1}^{n} a_{i j}(s)\left|\left(u_{j}\right)_{s}\right|_{C\left([-\tau, 0], X_{j}\right)}+c_{i}(s)\right) d s \\
& =\int_{0}^{t}\left(\sum_{j=1}^{n} a_{i j}(s)\left|u_{j}\right|_{C\left([s-\tau, s], X_{j}\right)}+c_{i}(s)\right) d s .
\end{aligned}
$$


Since $0 \leq s \leq t \leq a,\left|u_{j}\right|_{C\left([s-\tau, s], X_{j}\right)} \leq\left|u_{j}\right|_{C\left([-\tau, a], X_{j}\right)}$. Then

$$
\int_{0}^{t}\left|F_{i}\left(s, u_{s}\right)\right|_{X_{i}} d s \leq \sum_{j=1}^{n}\left|a_{i j}\right|_{L^{1}(0, a)}\left|u_{j}\right|_{C\left([-\tau, a], X_{j}\right)}+\left|c_{i}\right|_{L^{1}(0, a)} .
$$

Hence for $t \in[-\tau, a]$,

$$
\left|N_{i}(u)(t)\right|_{X_{i}} \leq \sum_{j=1}^{n}\left(\left|a_{i j}\right|_{L^{1}(0, a)}+b_{i j}\right)\left|u_{j}\right|_{C\left([-\tau, a], X_{j}\right)}+\gamma_{i},
$$

where $\gamma_{i}=\left|c_{i}\right|_{L^{1}(0, a)}+d_{i}$. As a result, for every $i \in J$, one has the following estimation of the first component from the definition of the norm $\left|N_{i}(u)\right|_{\tau}$,

$$
\left|N_{i}(u)\right|_{C\left([-\tau, a], X_{i}\right)} \leq \sum_{j=1}^{n} m_{i j}\left|u_{j}\right|_{C\left([-\tau, a], X_{j}\right)}+\gamma_{i} .
$$

Furthermore, we estimate $\left|N_{i}(u)\right|_{C_{\theta}\left([a-\tau, T], X_{i}\right)}$. To this aim, let $t \in[a, T]$. One has

$$
\left|N_{i}(u)_{t}\right|_{C\left([-\tau, 0], X_{i}\right)}=\left|N_{i}(u)\right|_{C\left([t-\tau, t], X_{i}\right)} .
$$

Take any $s \in[t-\tau, t]$ and try to estimate $\left|N_{i}(u)(s)\right|_{X_{i}}$. For $s \leq a$, we already have the estimation given by (3.9). Let $s \in[a, t]$. Then

$$
\begin{aligned}
\left|N_{i}(u)(s)\right|_{X_{i}} & \leq\left|\alpha_{i}(u)(0)\right|_{X_{i}}+\int_{0}^{a}\left|F_{i}\left(\xi, u_{\xi}\right)\right|_{X_{i}} d \xi+\int_{a}^{s}\left|F_{i}\left(\xi, u_{\xi}\right)\right|_{X_{i}} d \xi \\
& \leq \sum_{j=1}^{n} m_{i j}\left|u_{j}\right|_{C\left([-\tau, a], X_{j}\right)}+\gamma_{i}+\int_{a}^{s}\left|F_{i}\left(\xi, u_{\xi}\right)\right|_{X_{i}} d \xi
\end{aligned}
$$

Also

$$
\begin{aligned}
\int_{a}^{s}\left|F_{i}\left(\xi, u_{\xi}\right)\right|_{X_{i}} d \xi & \leq \int_{a}^{s}\left(\sum_{j=1}^{n} a_{i j}(\xi)\left|\left(u_{j}\right)_{\xi}\right|_{C\left([-\tau, 0], X_{j}\right)}+c_{i}(\xi)\right) d \xi \\
& \leq \sum_{j=1}^{n} \int_{a}^{s} a_{i j}(\xi)\left|\left(u_{j}\right)_{\xi}\right|_{C\left([-\tau, 0], X_{j}\right)} d \xi+\left|c_{i}\right|_{L^{1}(a, T)}
\end{aligned}
$$

Now we estimate the integral terms

$$
\begin{aligned}
\int_{a}^{s} a_{i j}(\xi)\left|\left(u_{j}\right)_{\xi}\right|_{C\left([-\tau, 0], X_{j}\right)} d \xi & =\int_{a}^{s} a_{i j}(t) e^{\theta(t-a)}\left|\left(u_{j}\right)_{t}\right|_{C\left([-\tau, 0], X_{j}\right)} e^{-\theta(t-a)} d t \\
& \leq\left|u_{j}\right|_{C_{\theta}\left([a-\tau, T], X_{j}\right)} \int_{a}^{s} a_{i j}(t) e^{\theta(t-a)} d t .
\end{aligned}
$$

Let $q$ by the conjugate exponent of $p$, i.e., $1 / p+1 / q=1$. Using Hölder's inequality,

$$
\int_{a}^{s} a_{i j}(t) e^{\theta(t-a)} d t \leq \frac{1}{(q \theta)^{1 / q}}\left|a_{i j}\right|_{L^{p}(a, T)} e^{\theta(s-a)},
$$


one can continue the estimation in (3.10) and obtain

$$
\left|N_{i}(u)(s)\right|_{X_{i}} \leq e^{\theta(s-a)} \sum_{j=1}^{n}\left(m_{i j}+\frac{1}{(q \theta)^{1 / q}}\left|a_{i j}\right|_{L^{p}(a, T)}\right)\left|u_{j}\right|_{\tau}+\widetilde{\gamma}_{i},
$$

where $\widetilde{\gamma}_{i}=\gamma_{i}+\left|c_{i}\right|_{L^{1}(a, T)}=\left|c_{i}\right|_{L^{1}(0, a)}+d_{i}$. Taking the maximum for $s \in[t-\tau, t]$ yields

$$
\left|N_{i}(u)_{t}\right|_{C\left([-\tau, 0], X_{i}\right)} \leq e^{\theta(t-a)} \sum_{j=1}^{n}\left(m_{i j}+\frac{1}{(q \theta)^{1 / q}}\left|a_{i j}\right|_{L^{p}(a, T)}\right)\left|u_{j}\right|_{\tau}+\widetilde{\gamma}_{i} .
$$

Dividing by $e^{-\theta(t-a)}$ and taking the maximum for $t \in[a, T]$ gives

$$
\left|N_{i}(u)\right|_{C_{\theta}\left([a-\tau, T], X_{i}\right)} \leq \sum_{j=1}^{n} \widetilde{m}_{i j}\left|u_{j}\right|_{\tau}+\widetilde{\gamma}_{i}
$$

where

$$
\widetilde{m}_{i j}=m_{i j}+\frac{1}{(q \theta)^{1 / q}}\left|a_{i j}\right|_{L^{p}(a, T)} .
$$

This together with (3.9) proves that

$$
\left|N_{i}(u)\right|_{\tau} \leq \sum_{j=1}^{n} \widetilde{m}_{i j}\left|u_{j}\right|_{\tau}+\widetilde{\gamma}_{i}, \quad i \in J
$$

or under the matricial form

$$
\|N(u)\| \leq \widetilde{M}\|u\|+\widetilde{\gamma}
$$

where $\widetilde{M}$ is the $n \times n$ matrix $\left[\widetilde{m}_{i j}\right]$, and $\widetilde{\gamma}$ is the column vector $\left(\widetilde{\gamma}_{1}, \ldots, \widetilde{\gamma}_{n}\right)^{t r}$. Since the spectral radius of the matrix $M$ is assumed less than one, and the elements of the matrix $\widetilde{M}$ can be assumed as close to the elements of $M$ as we wish (by taking a sufficiently large $\theta>0$ ), from Proposition 2.3 we may consider that the spectral radius of $\widetilde{M}$ is less than one. The proof is finished if there is a vector $R=\left(R_{1}, \ldots, R_{n}\right)^{t r}$ of nonnegative numbers such that

$$
\widetilde{M} R+\widetilde{\gamma} \leq R
$$

that is $(I-\widetilde{M}) R \geq \widetilde{\gamma}$. Indeed we can take $R=(I-\widetilde{M})^{-1} \widetilde{\gamma}$ whose components are nonnegative since the matrix $I-\widetilde{M}$ is inverse-positive.

Proof of Lemma 3.3. Similar estimations to those in the proof of Lemma 3.2 give for $i \in J_{1}$ and any $u, v \in C([-\tau, T], X)$,

$$
\left|N_{i}(u)-N_{i}(v)\right|_{C\left([-\tau, a], X_{i}\right)} \leq \sum_{j=1}^{n} m_{i j}\left|u_{j}-v_{j}\right|_{C\left([-\tau, a], X_{j}\right)}
$$


and

$$
\left|N_{i}(u)-N_{i}(v)\right|_{C_{\theta}\left([a-\tau, T], X_{i}\right)} \leq \sum_{j=1}^{n} \widetilde{m}_{i j}\left|u_{j}-v_{j}\right|_{\tau}
$$

Hence

$$
\left|N_{i}(u)-N_{i}(v)\right|_{\tau} \leq \sum_{j=1}^{n} \widetilde{m}_{i j}\left|u_{j}-v_{j}\right|_{\tau}
$$

Since for $i \in J_{2}$ the component $A_{i}$ of the operator $A$ are zero, Lipschitz inequalities as above also hold with all the Lipschitz constants equal to zero. Consequently,

$$
\|A(u)-A(v)\| \leq \widehat{M}\|u-v\|
$$

where $\widehat{M}$ is the $n \times n$ square matrix $\left[\widehat{m}_{i j}\right]$, with

$$
\widehat{m}_{i j}= \begin{cases}\widetilde{m}_{i j} & \text { for } i \in J_{1}, j \in J \\ 0 & \text { for } i \in J_{2}, j \in J\end{cases}
$$

Clearly $\widehat{M} \leq \widetilde{M}$, hence according to Proposition 2.4, the spectral radius of $\widehat{M}$ is less than one. Then (3.11) shows that $A$ is a generalized contraction in Perov's sense.

Proof of Lemma 3.4. The first components $B_{i}$ for $i \in J_{1}$ are zero, so compact. The continuity and growth conditions for $F_{i}\left(i \in J_{2}\right)$ make that the mappings $u \mapsto F$ ${ }_{i}\left(t, u_{t}\right)$ are continuous and bounded (send bounded sets into bounded sets). Also from (H4), the operators $\alpha_{i}$ and $S_{i}(t)$ are compact for $i \in J_{2}$ and $t>0$. Then the generalized version of the Arzelà-Ascoli theorem for functions with values in a metric space (see, e.g., [34, pp 72-74] and [43, p 296]), guarantees that the operator $B_{i}$ is completely continuous for every $i \in J_{2}$.

For the next result, none of the mappings $\alpha_{i}$ is assumed to be completely continuous.

Theorem 3.5. Assume that the conditions (H1), $\left(\mathrm{H} 2{ }^{*}\right)$ and $\left(\mathrm{H} 3^{*}\right)$ hold for every $i \in J_{1}$, and that $\left(\mathrm{H} 1^{*}\right),(\mathrm{H} 2)$ and $\left(\mathrm{H} 3^{*}\right)$ hold for every $i \in J_{2}$. In addition assume that the spectral radius of the $n \times n$ square matrix $M=\left[m_{i j}\right]$, where

$$
m_{i j}:=\left|a_{i j}\right|_{L^{1}(0, a)}+b_{i j}
$$

is less than one. Then the problem (1.1) has at least one mild solution on $[-\tau, T]$. In case that $m=n$, the solution is unique.

Proof. The only one difference is the way that the operator $N$ is split as $N=A+B$. Now we take

$$
A=\left(N_{1}, \ldots, N_{m}, A_{m+1}, \ldots, A_{n}\right), \quad B=\left(0, \ldots, 0, B_{m+1}, \ldots, B_{n}\right),
$$


where for $i \in J_{2}$,

$$
A_{i}(u)(t)= \begin{cases}\alpha_{i}(u)(t), & t \in[-\tau, 0] \\ S_{i}(t) \alpha_{i}(u)(0), & t \in[0, T]\end{cases}
$$

and

$$
B_{i}(u)(t)= \begin{cases}0, & t \in[-\tau, 0], \\ \int_{0}^{t} S_{i}(t-s) F_{i}\left(s, u_{s}\right) d s, & t \in[0, T] .\end{cases}
$$

The operator $B$ is completely continuous, while $A$ satisfies

$$
\|A(u)-A(v)\| \leq \widehat{M}^{0}\|u-v\|,
$$

where $\widehat{M}^{0}$ is the $n \times n$ square matrix $\left[\widehat{m}_{i j}^{0}\right]$, with

$$
\widehat{m}_{i j}^{0}= \begin{cases}\widetilde{m}_{i j} & \text { for } i \in J_{1}, j \in J \\ b_{i j} & \text { for } i \in J_{2}, j \in J\end{cases}
$$

which also satisfies $\widehat{M}^{0} \leq \widetilde{M}$.

We conclude by two examples illustrating our main result.

Example 1. Consider the semilinear delay transport system

$$
\begin{cases}u_{1}^{\prime}(t)+d^{1} \cdot \nabla u_{1}(t)=\alpha_{11}(t) u_{1}(t-\tau)+\alpha_{12}(t) \sin u_{2}(t-\tau), & t \in[0, T], \\ u_{2}^{\prime}(t)+d^{2} \cdot \nabla u_{2}(t)=\alpha_{21}(t) \cos u_{1}(t-\tau)+\alpha_{22}(t) u_{2}(t-\tau), & t \in[0, T], \\ u_{1}(t)=\int_{0}^{a}\left(\beta_{11}(t) u_{1}(t+s)+\beta_{12}(t) u_{2}(t+s)\right) d s, & t \in[-\tau, 0], \\ u_{2}(t)=\int_{0}^{a}\left(\beta_{21}(t) u_{1}(t+s)+\beta_{22}(t) u_{2}(t+s)\right) d s, & t \in[-\tau, 0] .\end{cases}
$$

Here, $d^{1}, d^{2} \in \mathbb{R}^{n} ; \alpha_{i j} \in C[0, T]$ and $\beta_{i j} \in L^{1}(-\tau, 0)$, for $i, j=1,2 ; a \in[0, T] ;$ and $u_{i}(t)(x)=u_{i}(t, x)$ for $x \in \mathbb{R}^{n}, i=1,2$. Also, by a notation like $\sin u_{2}(t-\tau)$ we mean the function $x \mapsto \sin \left(u_{2}(t-\tau, x)\right)$.

Let us consider $X_{i}=L^{r_{i}}\left(\mathbb{R}^{n}\right)$, with $1 \leq r_{i}<\infty$ for $i=1,2$. Then (see [43, $\mathrm{p}$ 88]), the operators $A_{i}(i=1,2)$ defined by

$$
\begin{aligned}
D\left(A_{i}\right) & =\left\{v \in X_{i}: d^{i} \cdot \nabla v \in X_{i}\right\} \\
A_{i} v & =-d^{i} \cdot \nabla v=-\sum_{j=1}^{n} d_{j}^{i} \frac{\partial v}{\partial x_{j}},
\end{aligned}
$$

are generators of the $C_{0}$-groups of isometries $\left\{S_{i}(t) ; t \in \mathbb{R}\right\}$,

$$
S_{i}(t) v(x)=v\left(x-t d^{i}\right), \quad \text { for } v \in X_{i}, t \in \mathbb{R}, x \in \mathbb{R}^{n} .
$$

In this case all the assumptions of Theorem 3.1 are satisfied with $J=J_{1}=\{1,2\}$, $J_{2}=\emptyset, a_{i j}(t)=\left|\alpha_{i j}(t)\right|$ (absolute value) and $b_{i j}=\left|\beta_{i j}\right|_{L^{1}(-\tau, 0)}$. Therefore, if the 
spectral radius of the matrix

$$
M=\left[\begin{array}{cc}
\left|\alpha_{11}\right|_{L^{1}(0, a)}+\left|\beta_{11}\right|_{L^{1}(-\tau, 0)} & \left|\alpha_{12}\right|_{L^{1}(0, a)}+\left|\beta_{12}\right|_{L^{1}(-\tau, 0)} \\
\left|\alpha_{21}\right|_{L^{1}(0, a)}+\left|\beta_{21}\right|_{L^{1}(-\tau, 0)} & \left|\alpha_{22}\right|_{L^{1}(0, a)}+\left|\beta_{22}\right|_{L^{1}(-\tau, 0)}
\end{array}\right]
$$

is less than one, then the problem (3.13) has a unique mild solution in $C([-\tau, T]$, $\left.L^{r_{1}}\left(\mathbb{R}^{n}\right) \times L^{r_{2}}\left(\mathbb{R}^{n}\right)\right)$.

Example 2. Let us consider a semilinear reaction-diffusion system of two MackeyGlass type diffusive equations, with multi-point nonlocal initial conditions

$$
\begin{cases}\frac{\partial u}{\partial t}(t, x)-d_{1} \Delta u(t, x)=f\left(t, u_{t}, v_{t}\right) u(t-\tau, x)-\lambda_{1} u(t, x)-a_{12}(t) v(t, x), & \text { in } Q, \\ \frac{\partial v}{\partial t}(t, x)-d_{2} \Delta v(t, x)=g\left(t, u_{t}, v_{t}\right) v(t-\tau, x)-\lambda_{2} v(t, x)-a_{21}(t) u(t, x), & \text { in } Q, \\ \frac{\partial u}{\partial \nu}(t, x)=\frac{\partial v}{\partial \nu}(t, x)=0, & \text { on } \Sigma, \\ u(t, x)=\varphi(t)(x)+\sum_{k=1}^{p_{1}} \alpha_{1 k} u\left(t_{1 k}+t, x\right), & \text { in } Q_{\tau}, \\ v(t, x)=\psi(t)(x)+\sum_{j=k}^{p_{2}} \alpha_{2 k} v\left(t_{2 k}+t, x\right), & \text { in } Q_{\tau},\end{cases}
$$

where $Q=[0, T] \times \Omega, \Sigma=[0, T] \times \partial \Omega, Q_{\tau}=[-\tau, 0] \times \Omega, \Omega \subset \mathbb{R}^{n}$ is a smooth bounded domain, $d_{1}, d_{2}, \lambda_{1}, \lambda_{2}>0, \tau \geq 0$ and $0<t_{i 1}<\ldots<t_{i p_{i}} \leq T$ for $i=1,2$.

We assume that $f, g:[0, T] \times C\left([-\tau, 0], L^{2}(\Omega) \times L^{2}(\Omega)\right) \rightarrow \mathbb{R}$ are continuous; $\varphi$, $\psi \in C\left([-\tau, 0], L^{2}(\Omega)\right) ;|f(t, u, v)| \leq a_{11}(t), \quad|g(t, u, v)| \leq a_{22}(t)$ for all $t \in[0, T]$ and $u, v \in C[-\tau, 0]$; and that $a_{i j} \in L^{1}\left(0, T ; \mathbb{R}_{+}\right)$with $\left.a_{i j}\right|_{[0, a]} \in L^{p}(0, a)$ for some $p>1$, where $a=\max \left\{t_{i j}: j=1, \ldots, p_{i} ; i=1,2\right\}$.

We apply Theorem 3.5 with $X_{1}=X_{2}=L^{2}(\Omega)$, and to the operators $A_{i}$ : $D\left(A_{i}\right) \rightarrow L^{2}(\Omega)(i=1,2)$ given by

$$
\begin{aligned}
D\left(A_{i}\right) & =\left\{u \in H^{2}(\Omega): \frac{\partial u}{\partial \nu}=0 \text { on } \partial \Omega\right\}, \\
A_{i} u & =d_{i} \Delta u-\lambda_{i} u,
\end{aligned}
$$

which generate compact semigroups [11, Theorem 1.11.8]. Here $J_{1}=\emptyset$ and $J_{2}=J=$ $\{1,2\}, b_{i i}=\sum_{k=1}^{p_{i}}\left|\alpha_{i k}\right|(i=1,2)$ and $b_{12}=b_{21}=0$. Therefore, if the spectral radius of the matrix

$$
M=\left[\left|a_{i j}\right|_{L^{1}(0, a)}+b_{i j}\right]_{i, j=1,2}
$$

is less than one, then the problem (3.14) has at least one mild solution in $C([-\tau, T]$, $\left.L^{2}(\Omega) \times L^{2}(\Omega)\right)$.

\section{ACKNOWLEDGEMENTS}

This work was supported by a grant of the Romanian National Authority for Scientific Research, CNCS-UEFISCDI, project number PN-II-ID-PCE-2011-3-0094 and by a 
grant of the Babeş-Bolyai University of Cluj-Napoca, through GTC-2016, project number 31782, project title "Operator methods for nonlinear systems with nonlocal conditions and applications".

\section{REFERENCES}

[1] A. Berman, R.J. Plemmons, Nonnegative Matrices in the Mathematical Sciences, SIAM, Philadelphia, 1994.

[2] O. Bolojan-Nica, G. Infante, P. Pietramala, Existence results for impulsive systems with initial nonlocal conditions, Math. Model. Anal., 18 (2013), 599-611.

[3] O. Bolojan, R. Precup, Implicit first order differential systems with nonlocal conditions, Electron. J. Qual. Theory Differ. Equ. 69 (2014), 1-13.

[4] O. Bolojan, R. Precup, Semilinear evolution systems with nonlinear constraints, Fixed Point Theory 17 (2016), 275-288.

[5] O. Bolojan-Nica, G. Infante, R. Precup, Existence results for systems with coupled nonlocal initial conditions, Nonlinear Anal. 94 (2014), 231-242.

[6] O. Bolojan, G. Infante, R. Precup, Existence results for systems with coupled nonlocal nonlinear initial conditions, Math. Bohem. 140 (2015), 371-384.

[7] A. Boucherif, H. Akca, Nonlocal Cauchy problems for semilinear evolution equations, Dynam. Systems Appl. 11 (2002), 415-420.

[8] A. Boucherif, R. Precup, On the nonlocal initial value problem for first order differential equations, Fixed Point Theory 4 (2003), 205-212.

[9] A. Boucherif, R. Precup, Semilinear evolution equations with nonlocal initial conditions, Dynam. Systems Appl. 16 (2007), 507-516.

[10] M. Burlică, D. Roşu, I.I. Vrabie, Abstract reaction-diffusion systems with nonlocal initial conditions, Nonlinear Anal. 94 (2014), 107-119.

[11] M.-D. Burlică, M. Necula, D. Roşu, I.I. Vrabie, Delay Differential Evolutions Subjected to Nonlocal Initial Conditions, Chapman and Hall/CRC Press, 2016.

[12] L. Byszewski, Theorems about the existence and uniqueness of solutions of semilinear evolution nonlocal Cauchy problems, J. Math. Anal. Appl. 162 (1991), 494-505.

[13] T. Cardinali, R. Precup, P. Rubbioni, A unified existence theory for evolution equations and systems under nonlocal conditions, J. Math.Anal.Appl. 432 (2015), 1039-1057.

[14] J. Chabrowski, On nonlocal problems for parabolic equations, Nagoya Math. J. 93 (1984), 109-131. 
[15] T. Cazenave, A. Haraux, An Introduction to Semilinear Evolution Equations, Oxford University Press, New York, 1998.

[16] N. Cioranescu, Sur les conditions linéaires dans l'intégration des équations différentielles ordinaires, Math. Z. 35 (1932), 601-608.

[17] R. Conti, Recent trends in the theory of boundary value problems for ordinary differential equations, Boll. Un. Mat. Ital. 22 (1967), 135-178.

[18] K. Deng, Exponential decay of solutions of semilinear parabolic equations with initial boundary conditions, J. Math. Anal. Appl. 179 (1993), 630-637.

[19] J. García-Falset, S. Reich, Integral solutions to a class of nonlocal evolution equations, Commun. Contemp. Math. 12 (2010), 1032-1054.

[20] J.A. Goldstein, Semigroups of Linear Operators and Applications, Oxford University Press, New York, 1985.

[21] G. Infante, M. Maciejewski, Multiple positive solutions of parabolic systems with nonlinear, nonlocal initial conditions, submitted.

[22] D. Jackson, Existence and uniqueness of solutions to semilinear nonlocal parabolic equations, J. Math. Anal. Appl. 172 (1993), 256-265.

[23] G.L. Karakostas, P.Ch. Tsamatos, Existence of multiple positive solutions for a nonlocal boundary value problem, Topol. Methods Nonlinear Anal. 19 (2002), $109-121$.

[24] A.A. Kerefov, Nonlocal boundary value problems for parabolic equations (Russian), Differ. Uravn. 15 (1979), 74-78.

[25] V. Kolmanovskii, A. Myshkis, Applied Theory of Functional Differential Equations, Kluwer, Dordrecht, 1992.

[26] Y. Lin, J.H. Liu, Semilinear integrodifferential equations with nonlocal Cauchy problem, Nonlinear Anal. 26 (1996), 1023-1033.

[27] J.H. Liu, A remark on the mild solutions of non-local evolution equations, Semigroup Forum 66 (2003), 63-67.

[28] M. McKibben, Discovering Evolution Equations with Applications, Vol. I, Chapman \&Hall/CRC, 2011.

[29] M. Necula, I.I. Vrabie, Nonlinear delay evolution inclusions with general nonlocal initial conditions, Ann. Acad. Rom. Sci. Ser. Math. 7 (2015), 67-97.

[30] O. Nica, Initial-value problems for first-order differential systems with general nonlocal conditions, Electron. J. Differential Equations 2012 (2012), no. 74, 1-15.

[31] O. Nica, R. Precup, On the nonlocal initial value problem for first order differential systems, Stud. Univ. Babeş-Bolyai Math. 56 (2011), no. 3, 125-137. 
[32] S.K. Ntouyas, P.Ch. Tsamatos, Global existence for semilinear evolution equations with nonlocal conditions, J. Math. Anal. Appl. 210 (1997), 679-687.

[33] W.E. Olmstead, C.A. Roberts, The one-dimensional heat equation with a nonlocal initial condition, Appl. Math. Lett. 10 (1997), 89-94.

[34] D. O'Regan, R. Precup, Theorems of Leray-Schauder Type and Applications, Gordon and Breach, Amsterdam, 2001.

[35] A. Paicu, I.I. Vrabie, A class of nonlinear evolution equations subjected to nonlocal initial conditions, Nonlinear Anal. 72 (2010), 4091-4100.

[36] P. Pietramala, A note on a beam equation with nonlinear boundary conditions, Bound. Value Probl. 2011, Art. ID 376782, 14 pp.

[37] C. V. Pao, Reaction diffusion equations with nonlocal boundary and nonlocal initial conditions, J. Math. Anal. Appl. 195 (1995), 702-718.

[38] R. Precup, The role of matrices that are convergent to zero in the study of semilinear operator systems, Math. Comp. Modelling 49 (2009), 703-708.

[39] R. Precup, D. Trif, Multiple positive solutions of non-local initial value problems for first order differential systems, Nonlinear Anal. 75 (2012), 5961-5970.

[40] A. Štikonas, A survey on stationary problems, Green's functions and spectrum of Sturm-Liouville problem with nonlocal boundary conditions, Nonlinear Anal. Model. Control 19 (2014), 301-334.

[41] P.N. Vabishchevich, Non-local parabolic problems and the inverse heat-conduction problem (Russian), Differ. Uravn. 17 (1981), 761-765.

[42] A. Viorel, Contributions to the Study of Nonlinear Evolution Equations, Ph.D. Thesis, Cluj-Napoca, 2011.

[43] I.I. Vrabie, $C_{0}$-Semigroups and Applications, Elsevier, Amsterdam, 2003.

[44] I.I. Vrabie, Global solutions for nonlinear delay evolution inclusions with nonlocal initial conditions, Set-Valued Var. Anal. 20 (2012), 477-497.

[45] J.R.L. Webb, G. Infante, Positive solutions of nonlocal initial boundary value problems involving integral conditions, NoDEA Nonlinear Differential Equations Appl. 15 (2008), 45-67.

[46] W. M. Whyburn, Differential equations with general boundary conditions, Bull. Amer. Math. Soc. 48 (1942), 692-704. 